\title{
Precision measurements at the LHC
}

\section{Simone Amoroso*, on behalf of the ATLAS and CMS Collaborations}

Deutsches Elektronen-Synchrotron, Notkestraße 85, 22607 Hamburg, Germany

E-mail: simone.amoroso@desy.de

This contribution highlights selected results from the programme of precision Standard Model measurements of the ATLAS and CMS Collaboration at the Large Hadron Collider (LHC). The measurements presented use data from $p p$ collisions at $\sqrt{s}=7$ and $8 \mathrm{TeV}$ collected during the LHC Run-1, as well as first results using data at centre of mass energy $\sqrt{s}=13 \mathrm{TeV}$ in LHC Run-2. These include measurements of the electroweak mixing angle, of the top-quark and Higgs boson masses, and a first LHC measurement of the mass of the $W$-bosons. Perspective for precision measurements at future High Luminosity runs of the LHC are also briefly discussed.

14th International Symposium on Radiative Corrections (RADCOR2019)

9-13 September 2019

Palais des Papes, Avignon, France

${ }^{*}$ Speaker. 


\section{Introduction}

Measuring the parameters of the Standard Model (SM) to very high precision is a pillar of the physics programme of the Large Hadron Collider (LHC) [1]. With the measurement of $M_{H}$ the electroweak sector of the SM is overconstrained and global fits can predict key observables, such as the $W$-boson mass and the effective electroweak mixing angle, with a precision exceeding that of the direct measurement [4]. The comparison of measured and predicted values thus provides a crucial test of the SM, and might hint at possible physics beyond the SM. After an astounding Run1 with $8 \mathrm{TeV}$ proton-proton collisions featuring among others the discovery of the Higgs boson, the Run- 2 of the Large Hadron Collider (LHC) has started in 2015 colliding protons with unprecedented $13 \mathrm{TeV}$ centre-of-mass energy. The high collision energy and the large datasets collected allow the exploration of less well tested areas of the SM and to improve significantly on the precision of many SM parameters. This contribution gives an overview of precision SM measurements of electroweak parameters performed by the ATLAS [2] and CMS [3] experiments at the LHC, with a focus on the main theoretical uncertainties and on prospects for future measurements.

\section{The W-boson mass}

The determination of the $W$-boson mass from the global fit of electroweak parameters has an uncertainty of $8 \mathrm{MeV}$ [4], which sets a natural target for the precision of experimental measurements. The current world average, driven by the Tevatron measurements, has a $15 \mathrm{MeV}$ uncertainty. Measuring the $W$ mass is particularly challenging at the LHC due to the large number of interactions per beam crossing and the contribution of strange and charm quarks parton distribution functions (PDFs) to $W$ production. The ATLAS Collaboration has performed the first LHC measurement of the $W$-boson mass in $W \rightarrow e v, \mu v$ decays using $4.7 \mathrm{fb}^{-1}$ of data collected at $\sqrt{s}=7 \mathrm{TeV}$ during the LHC Run-1 [5]. The measurement is based on template fits to the distributions of the charged lepton transverse momentum $p_{T}^{l}$ and the transverse mass of the $W$-boson candidate $m_{T}=\sqrt{(1-\cos \Delta \phi)}$, where $p_{T}^{\text {pmiss }}=\left(p_{T}^{l}+u_{T}^{l}\right)$ is the missing transverse momentum and $\Delta \phi$ is the azimuthal opening angle between the charged lepton and the missing transverse momentum. The recoil in the transverse plane, $u_{T}$, is constructed from the vector sum of the transverse energy of all clusters reconstructed in the calorimeters, excluding those from the charged lepton. The $p_{T}^{l}$ distribution is sensitive to the modelling of the transverse momentum of the $W$ boson $\left(p_{T}^{W}\right)$, while the $m_{T}$ distribution is primarily determined by the experimental resolution of the reconstructed $u_{T}$ and is sensitive to the modelling of the underlying event and of additional simultaneous $p p$ collisions. Both the experimental calibrations and the physics model rely heavily on control measurements of the neutral Drell-Yan process. The hadronic recoil and the leptons are calibrated in $Z \rightarrow l l$ events. The uncertainty on the $W$-boson transverse momentum is constrained using the very precise $7 \mathrm{TeV}$ measurement of the $Z$-boson $p_{T}$. PDF uncertainties are validated using precise measurement of the $Z$-rapidity distribution and the $W$-helicity fractions using an $8 \mathrm{TeV}$ measurement of angular coefficients in $Z$ events [14]. Excellent agreement is found among the $e / \mu$, $W^{+} / W^{-}$and $p_{T}^{l} / m_{T}$ channels and the final combined result achieves a precision of $0.02 \%$ :

$$
m_{W}=80370 \pm 7 \text { (stat.) } \pm 11 \text { (exp. syst.) } \pm 14 \text { (mod. syst.) } \mathrm{MeV}=80370 \pm 19 \mathrm{MeV}
$$


The result, shown in Fig. 1, is consistent with the SM expectation, compatible with the world average and competitive with the Tevatron combination. The precision is dominated by the $p_{T}^{l}$ measurement with large uncertainties from modelling effects. PDF uncertainties affect the $m_{W}$ measurement as they determine flavour and momentum of the incoming partons, which then impact the $W$ boson's rapidity, transverse momentum and polarisation. The modelling of $p_{T}^{W}$ is also a source of large uncertainties. State-of-the-art NNLO+NNLL predictions are found to disagree with data for the ratio of $p_{T}(W / Z)$. The $p_{T}^{W}$ spectrum is therefore determined by extrapolating $Z \rightarrow l l$ data using a parton shower (PS) predictions "tuned" to the measured $p_{T}^{Z}[6]$. This procedure yields an uncertainty on $p_{T}^{W}$ of $2.5 \%$ at low $p_{T}^{W}$, dominated by uncertainties on the modelling of the differences in the initial states of the $W$ - and $Z$-boson productions. The precision of direct measurements of $p_{T}^{W}$ is driven by the resolution on the hadronic recoil $\sigma\left(u_{T}\right)$, which strongly increases with the number of simultaneous $p p$ collisions (pile-up, $\langle\mu\rangle$ ). A CMS measurement of the $p_{T}^{W} / p_{T}^{Z}$ ratio at $\sqrt{s}=8 \mathrm{TeV}$ achieves a precision at the level of a few $\%$ [8]. ATLAS and CMS have collected about $380 \mathrm{pb}^{-1}$ and $260 \mathrm{pb}^{-1}$ of data at $\sqrt{s}=13 \mathrm{TeV}$, and $200 \mathrm{pb}^{-1}$ and $300 \mathrm{pb}^{-1}$ at $\sqrt{s}=5 \mathrm{TeV}$, respectively, both at the low pile-up of $\langle\mu\rangle \sim 2$. Such data will provide enough $W$-boson candidates to reach a $\sim 1 \%$ uncertainty on a direct measurement of $p_{T}^{W}$ and a $10 \mathrm{MeV}$ statistical uncertainty on $m_{W}$ [9]. Better knowledge of the PDFs, and improved QCD and electroweak predictions for Drell-Yan production, will remain crucial for future measurements of the $W$-boson mass at the LHC.
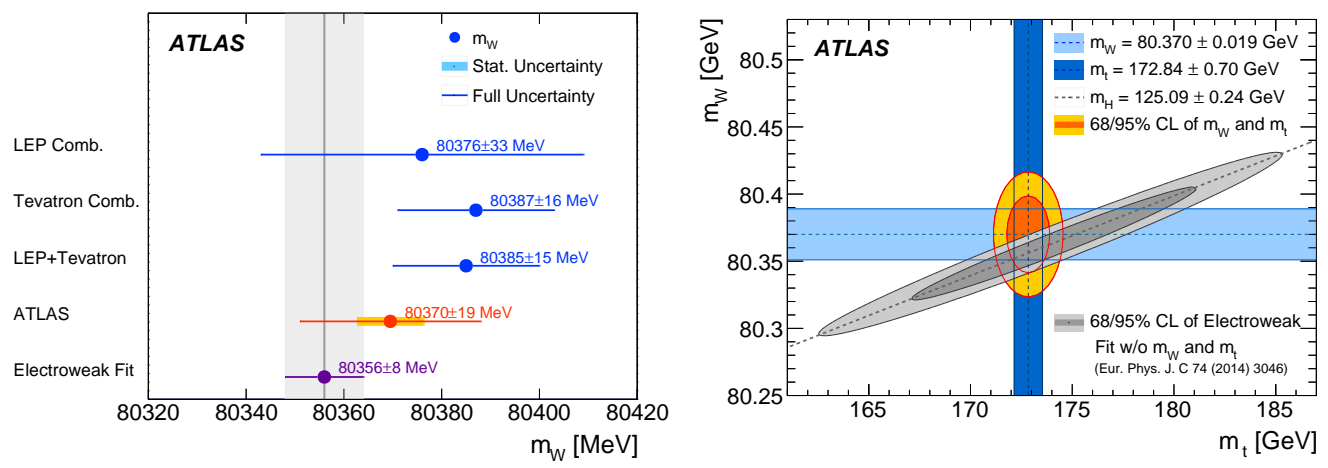

Figure 1: [left:] The ATLAS measured value of $m_{W}$ compared to other published results, including measurements from the LEP and Tevatron experiments and the prediction from the global electroweak fit. [right:] The $68 \%$ and $95 \%$ confidence-level contours of the $m_{W}$ and $m_{t}$ indirect determination from the global electroweak fit compared to the direct ATLAS measurements of the top-quark and $W$-boson masses [5].

\section{The effective leptonic weak mixing angle}

The weak mixing angle, $\sin ^{2} \theta_{W}$, is a fundamental SM parameter which relates the masses of the $W$ - and $Z$-bosons and determines their relative couplings to matter. It is most common experimentally to perform measurements of the effective weak mixing angle, $\sin ^{2} \theta_{f}^{\text {eff }}=k_{f} \cdot \sin ^{2} \theta_{W}$, which absorbs higher-order modifications from electroweak virtual and radiative corrections. The two most precise determinations at lepton colliders display a significant $(3.2 \sigma)$ tension among each 
other, making further measuremets very valuable. At the LHC, the effective leptonic weak mixing angle, $\sin ^{2} \theta_{l}^{\text {eff }}$, is measured from asymmetries in the angular distributions of the leptons from $Z \rightarrow e e$, decays. The ATLAS and CMS experiments measured $\sin ^{2} \theta_{\text {eff }}^{l}$ with $20.2 \mathrm{fb}^{-1}$ and up to $19.6 \mathrm{fb}^{-1}$ of $p p$ data collected at $\sqrt{s}=8 \mathrm{TeV}$, respectively, using different approaches. The CMS measurement of $\sin ^{2} \theta_{\text {eff }}^{l}$ [13] is based on a fiducial measurement of the forward-backward asymmetry, $A_{\mathrm{FB}}=\left(\sigma\left(\cos \theta^{*}>0\right)-\sigma\left(\cos \theta^{*}<0\right)\right) /\left(\sigma\left(\cos \theta^{*}>0\right)+\sigma\left(\cos \theta^{*}<0\right)\right)$, where $\theta^{*}$ is the polar angle of the negative lepton in the Collins-Soper frame. The measurement is performed in 12 bins in $m_{11}$ and 6 bins in $y_{11}$, and a fit to the measured $A_{\mathrm{FB}}$ with templates built from predictions at NLO+PS is used to derive $\sin ^{2} \theta_{\text {eff }}^{l}$. In the ATLAS measurement [15], $\sin ^{2} \theta_{\text {eff }}^{l}$ is extracted from a measurement of the $A_{4}$ angular coefficient, one of eight coefficients which, together with the unpolarised cross section, describe entirely the QCD dynamics of $Z$-boson production. The measurement is performed in the full phase space of the decay leptons in 10 bins in $m_{11}$ and $y_{11}$. From the $A_{4}$ measurement, $\sin ^{2} \theta_{\text {eff }}^{l}$ is derived using NNLO QCD predictions reweighted to include effects from NLO electroweak corrections. The ATLAS measurement includes events with one central electron $\left(\left|\eta_{l}\right|<2.4\right)$ and one forward electron $\left(2.5<\left|\eta_{l}\right|<4.9\right)$, giving access to high $\left|y_{11}\right|$ values. The sign of the asymmetry depends on the direction of the incoming quark, which in $p p$ collision is estimated from the direction of the reconstructed $Z$-boson, as the valence quark tends to carry a larger fraction of the proton momentum than the sea quark. In forward events with large $\left|y_{11}\right|$, this assignment is more accurate than in central events, with the ATLAS high- $\left|y_{11}\right| e e$ channel achieving better precision than the central- $\left|y_{11}\right| e e+\mu \mu$ combination. While the measurements of $A_{4}$ and $A_{\mathrm{FB}}$ are dominated by statistical uncertainties, the precision of the interpretations in terms of $\sin ^{2} \theta_{\text {eff }}^{l}$ are dominated by PDF uncertainties. To reduce the impact of the PDFs, correlations in the expected $A_{4}$ and $A_{\mathrm{FB}}$ as functions of $\left|y_{11}\right|$ and $m_{\mathrm{ll}}$ induced by PDF variations are used to constrain the PDF uncertainties in-situ to data. In the ATLAS measurement, PDF uncertainties are reduced through Hessian profiling, while in the CMS measurement a Bayesian $\chi^{2}$ reweighting is used. These equivalent methods yield significant reductions in the size of the PDF uncertainties; a factor of 2 for the CMS measurement. Yet, PDF uncertainties remain the dominant systematic. Moreover, the spread in central values of $\sin ^{2} \theta_{\text {eff }}^{l}$ measured using different PDF sets is found larger than the size of the PDF uncertainties for a given set. The two measurements result are:

$$
\begin{aligned}
& \text { ATLAS: } \sin ^{2} \theta_{\text {eff }}^{l}=0.23140 \pm 0.00021 \text { (stat.) } \pm 0.00024 \text { (PDF) } \pm 0.00016 \text { (syst.) } \\
& \text { CMS: } \sin ^{2} \theta_{\text {eff }}^{l}=0.23101 \pm 0.00036 \text { (stat.) } \pm 0.00031 \text { (PDF) } \pm 0.00024 \text { (syst.) }
\end{aligned}
$$

As shown Fig. 2, they are in agreement with the global electroweak fit, and have similar precision to that of the most precise LEP/SLC measurements, and to that of the combination of Tevatron results.

\section{4. $W$ and $Z$ cross-section measurements}

The ATLAS and CMS experiments have published a wealth of measurements of differential cross sections of $W$ - and $Z$-boson productions at different center-of-mass energies ( $\sqrt{s}=2.76,5$, 7,8 and $13 \mathrm{TeV}$ ) and as a function of different kinematic quantities. Thanks to the large statistics of the collected samples and the accurate knowledge of the detector performance, the precision 

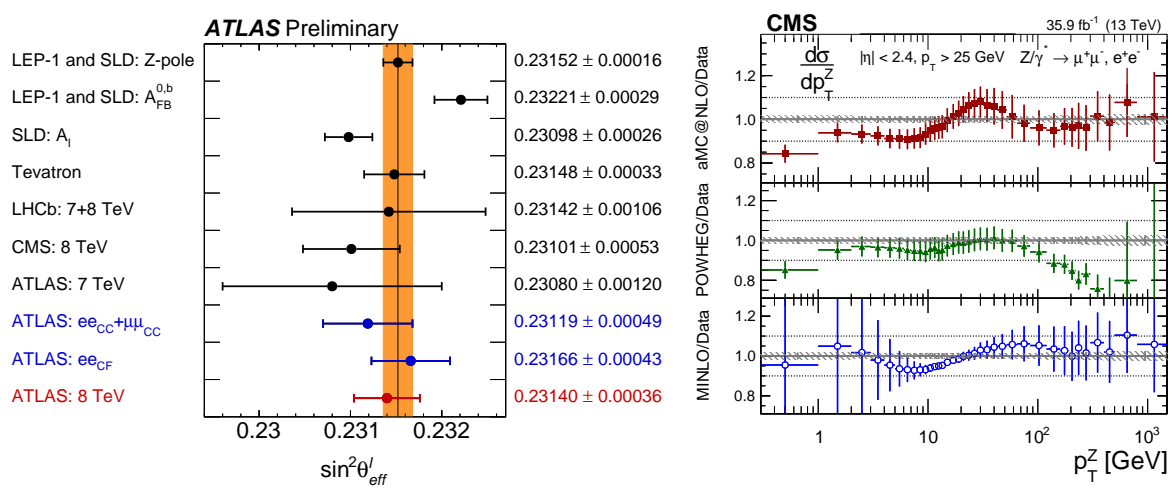

Figure 2: [left:] Comparison of the measurements of the effective leptonic weak mixing angle, $\sin ^{2} \theta_{l}^{\text {eff }}$, at LEP/SLC, the Tevatron and the LHC [15]. [right:] A comparison of a CMS measurement of $p_{T}^{Z}$ using $13 \mathrm{TeV}$ data against different Monte Carlo predictions [12].

of these measurements is significantly better than the precision of theoretical predictions. They thus provide crucial inputs to benchmark perturbative predictions and improve parton distribution functions needed for precise EW measurements. ATLAS measurements of $W$ and $Z$ cross-sections at $\sqrt{s}=7 \mathrm{TeV}$ reached an experimental precision of $0.6,0.5$, and $0.32 \%$ [10], with a total uncertainty only limited by the $1.8 \%$ uncertainty on the integrated luminosity. They have been shown to provide significant PDF constraints [11], and a preference for a large ratio of the strange-quark distribution to the lighter sea-quark distributions at low Bjorken- $x$. The CMS experiment released a $\sqrt{s}=13 \mathrm{TeV}$ measurement of the $Z$ production cross section differential in dilepton transverse momentum $p_{T}^{11}$, dilepton rapidity $\left|y^{11}\right|$ and $\phi^{*}$, a kinematic variable correlated to $\phi^{*} \sim p_{T}^{11} / m_{11}$ [12] . Absolute cross sections are measured in the detector fiducial volume $\left(\left|\eta_{l}\right|<2.4\right.$ and $\left.p_{T}^{l}>25 \mathrm{GeV}\right)$ using $35.9 \mathrm{fb}^{-1}$ of data and compared to a range of predictions, including Monte Carlo at NLO+PS accuracy, fixed order predictions at NNLO accuracy, and resummed predictions at NNLL accuracy. None of them is able to describe the data in the full $p_{T}^{Z}$ range as can be seen in2. Normalised cross sections are measured with uncertainties below $0.5 \%$ for $p_{T}^{11}<50 \mathrm{GeV}$, as these cross sections are not affected by the dominant uncertainty on the integrated luminosity.

\section{Top-quark cross-sections and mass}

As the heaviest particle in the SM, the top-quark plays an important role in electroweak symmetry breaking and is a sensitive probe for physics beyond the SM. Top-quark pair cross-sections have been measured at different energies, as shown in Fig. 3, they are in good agreement with predictions at NNLO including NNLL threshold resummation. A new measurement of the top-quark pair cross-section in the dilepton final state using $36.1 \mathrm{fb}^{-1}$ of $\sqrt{s}=13 \mathrm{TeV}$ data is ATLAS's most precise yet [17]. It is performed in dileptonic events, which despite accounting for only $2 \%$ of top-quark-pair decays, provide for a very clean sample of events with very little background. The $t \bar{t}$ production cross-section is extracted simultaneously to the efficiency to select, reconstruct, and $b$-tag a jet. This method allows for a very small jet systematics, at cost of large systematics from $t \bar{t} / W t$ interference in some phase space regions. The cross-section is determined to $2.4 \%$ precision, 
dominated by systematic uncertainties on the luminosity and beam energy.

$$
\sigma_{t \bar{t}}=826.4 \pm 3.6 \text { (stat.) } \pm 11.5 \text { (syst.) } \pm 15.7 \text { (lumi) } \pm 1.9 \text { (beam) pb }
$$

The result is combined with $\sqrt{s}=7 \mathrm{TeV}$ and $\sqrt{s}=8 \mathrm{TeV}$ measurements to derive ratios and double ratios of $t \bar{t}$ and $Z$ cross-sections at different energies sensitive to the PDF content of the proton. These ratios, shown in Fig. 3, are in good agreement with predictions from modern PDFs within two standard deviations, with the exception of ABM12 for the ratio of $t \bar{t} / Z$ cross-sections (attributed to lower gluon density at high Bjorken- $x$ in ABM12 compared to the other considered PDF sets). Kinematic distributions of the produced leptons also been precisely measured. These were compared to predictions from various Monte Carlo (MC) event generators highlighting several discrepancies, pointing to the need for more precise theoretical calculations of the $t \bar{t}$ process.
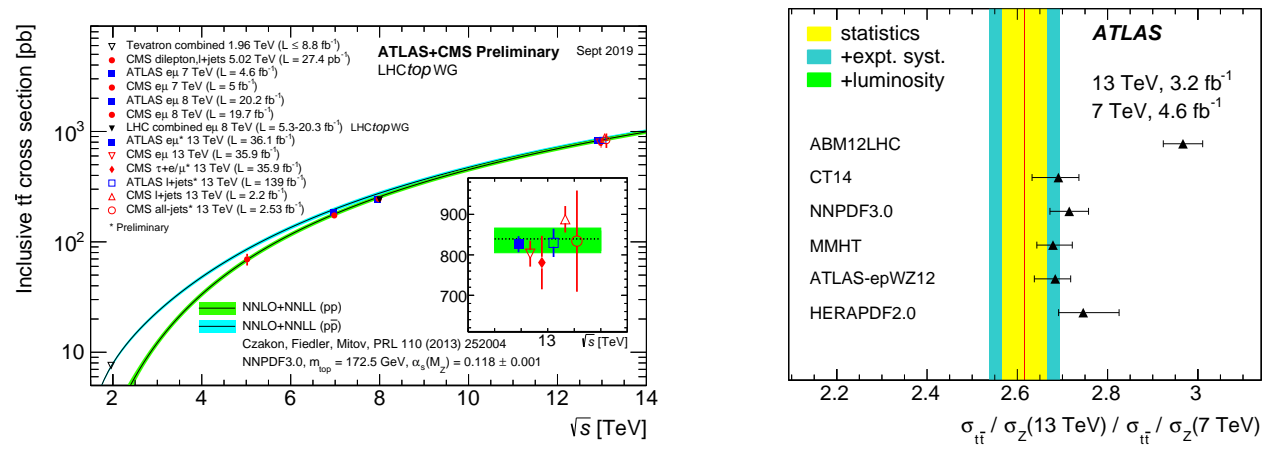

Figure 3: [left:] Summary of LHC and Tevatron measurements of the top-pair production cross-section as a function of the centre-of-mass energy compared to the NNLO QCD calculation complemented with NNLL resummation [16]. [right:] Double ratio of the $t \bar{t} / Z$ production cross-section at $\sqrt{s}=13$ and $7 \mathrm{TeV}$, compared with NNLO predictions interfaced to different PDF sets [17].

Top mass measurements at hadron colliders, as their precision approaches few hundred MeV's, poses difficult problems of theoretical interpretation, involving our understanding of non-perturbative effects in QCD, and of how they are implemented in shower generators. The currently most precise methods for top mass measurements at the LHC are from "direct measurements", obtained exploiting information from the kinematic reconstruction of the measured top quark decay products. In direct measurements, the measured top mass is interpreted as the value of the top mass parameter in the MC generator that is used (MC mass). A continuous campaign of measurements carried by ATLAS and CMS led to a precision for Run- 1 direct measurements of $<0.5 \mathrm{GeV}(<0.3 \%$ precision), as shown in Fig. 4. CMS performed a direct top mass mass measurement in the all-hadronic channel using $35.9 \mathrm{fb}^{-1}$ of $13 \mathrm{TeV}$ data [18]. It selects events with $\geq 6$ jets ( $\geq 2 b$-tagged), and requires $\sum p_{T}>450 \mathrm{GeV}$. It makes use of the "ideogram method", where a kinematic reconstruction of the $W b W b$ final state is followed by a fit to the $m_{t}$ and $m_{W}$ reconstructed masses to simultaneously extract the top mass and the a jet energy scale factor. The method achieves a precision of $m_{t}^{\mathrm{MC}}=172.34 \pm 0.20$ (stat+JSF) $\pm 0.43(\mathrm{CR}) \pm 0.55$ (syst.) $\mathrm{GeV}$, dominated by the systematics on the jet energy flavour response and by the modelling of non-perturbative colour reconnection effects in the MC generators. This latter systematic is larger than for Run-1 results, due to a comparison 
to a more comprehensive variety of colour reconnection models. A combination with results from the lepton+jets channel from the same dataset yields $m_{t}^{\mathrm{MC}}=172.26 \pm 0.61 \mathrm{GeV}$, with a relative uncertainty of $0.36 \%$.

Indirect mass measurements are an alternative approach which takes advantage of the top mass dependence of the production cross section by comparing measured cross-sections with theoretical calculations. In this case the precise value of the extracted mass will depend upon the renormalization scheme adopted in the theoretical prediction. ATLAS extracted the top mass from an $8 \mathrm{TeV}$ measurement of normalised differential cross section of top-quark pair production in association with an energetic jet in the lepton+jets final state [19]. The request of an additional jet enhancing the sensitivity to $m_{t}$. The observable used is $\rho_{0}=2 m_{0} / m(t \bar{t}+1$ jet $)$, with $m_{0}=170 \mathrm{GeV}$ and $m(t \bar{t}+1$ jet $)$ the invariant mass of the $t \bar{t} j$ system. The normalised parton-level cross-section is compared to NLO+PS QCD predictions to derive either the pole or the $\overline{M S}$ mass. The pole mass extraction yields $m_{t}^{\text {pole }}=171.1 \pm 0.4$ (stat.) \pm 0.9 (syst) \pm 0.7 (theo), dominated by experimental systematics. A similar procedure is used to extract the $\overline{M S}$ mass, which is found to be consistent with $m_{t}^{\text {pole }}$ when translated to the same scheme, but with larger uncertainties due to a larger scale dependence. CMS performed a measurement of $t \bar{t}$ cross-sections in dilepton events using $35.9 \mathrm{fb}^{-1}$ of $13 \mathrm{TeV}$ data. Normalised triple-differential cross-sections are measured as a function of the mass and rapidity of the $t \bar{t}$ system, and the number of additional jets. They are used for a simultaneous extraction of the top-quark mass and the strong coupling using NLO fixed-order theory [20], obtaining and $m_{t}$ (pole) $=170.5 \pm 0.8 \mathrm{GeV}$, dominated by uncertainties from the methodology and QCD scales. The data are also fitted together with the inclusive deep inelastic scattering data from HERA, determining simultaneously the gluon PDF, the strong coupling and $m_{t}^{\text {pole }}$, showing that their values depend only weakly on each other. This makes future PDF fits at NNLO, once corresponding theoretical predictions become available, very interesting. An ultimate precision on
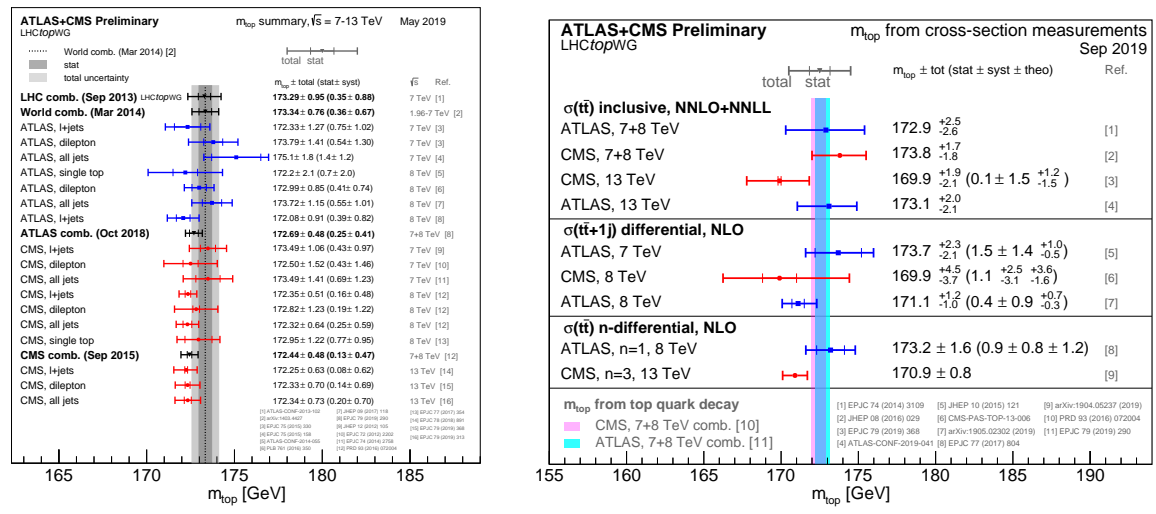

Figure 4: Summary of the ATLAS and CMS measurements of the top quark mass [left:] from top quark decay products ("direct") and [right:] from from $t \bar{t}$ production observables ("indirect") [16].

the top mass of few hundreds MeVs is within reach of the LHC Run-2 by combining measurements/experiments. But important work will need to be undertaken on reducing systematics, in particular those related to theory and signal modeling. Differences between $m_{t}$ theoretical definitions (pole mass, $\overline{M S}$ mass, MC mass) are also a fundamental issue to deal with as the experimental 
precision is approaching the expected size of these theoretical ambiguities.

\section{Higgs mass, cross-section, and couplings}

The number of recorded Higgs boson events has more than tripled since its discovery, allowing studies of the Higgs properties to enter the realm of precision physics. The mass of the Higgs boson, $m_{H}$ is the last SM parameter to fully determine the SM scalar sector. It is measured from mass peaks in the high-resolution $4 l$ and $\gamma \gamma$ channels, and its best determination is currently from the Run-1 ATLAS + CMS Higgs mass combination [21], which achieved a precision of $(0.2 \%)$ :

$$
m_{H}=125.09 \pm 0.24 \mathrm{GeV}= \pm 0.21 \text { (stat.) } \pm 0.11 \text { (syst.) } \mathrm{GeV}
$$

The precision of the measurement crucially relies on the performance in the determination of the photon and lepton energy scales, which are now known to a precision of about 2 per-mill. First Run-2 measurements of the $m_{H}$ have now been performed at $13 \mathrm{TeV}$. ATLAS combined $35.6 \mathrm{fb}^{-1}$ of data in the $\gamma \gamma$ and $4 l$ decay channels [22] obtaining $m_{H}=124.97 \pm 0.24 \mathrm{GeV}$, dominated by systematic uncertainties. CMS used the $4 l$ channel only, obtaining $m_{H}=125.26 \pm 0.21 \mathrm{GeV}$, with comparable statistical and systematic uncertainties [23]. The total Higgs production cross-section has now been measured to about $5 \%$ precision from the statistical combination of the high statistics channels $4 l$ and $\gamma \gamma$ [24]. Its value is in good agreement with state-of-the-art theory predictions at $\mathrm{N}^{3} \mathrm{LO}$ QCD + NLO EW. Fiducial differential cross-sections, and in particular $p_{T}^{H}$, are also found in good agreement with theory.

Measurement of the couplings properties of the Higgs boson are key to further understand the nature of the Higgs boson. A global analysis of the Higgs boson interactions with bosons and fermions is obtained by studying the production cross sections in several production modes and exploring different decay channels. Combined measurements of the Higgs boson couplings at $13 \mathrm{TeV}$ have been performed by ATLAS [26] using up to $80 \mathrm{fb}^{-1}$ of data and CMS [25] with $35.9 \mathrm{fb}^{-1}$ of data. The combinations are based on analyses of the five main Higgs boson production mechanisms (gluon fusion, vector boson fusion, and associated production with a $W$ or $Z$ boson, or a top quark-antiquark pair) and of different decay modes: $H \rightarrow \gamma \gamma, Z Z, W W, \tau \tau, b b$ and $\mu \mu$. Searches for invisible Higgs boson decays and measurements of off-shell Higgs boson production are also considered. All of the Higgs Yukawa are found agreement with SM expectations. The global signal strength is determined to be $\mu=1.11 \pm 0.08$ (ATLAS) and $\mu=1.17 \pm 0.10$ (CMS), assuming a Higgs boson mass of $125.09 \mathrm{GeV}$. The precision on the coupling measurements ranges from $7 \%$ to $15 \%$ including the fermion Yukawas, and independent measurements exists of the $b$, top and $\tau$ Yukawas. For the exception of the $H \rightarrow \mu \mu$ channel, systematic uncertainties are already dominating the measurement. Additionally, a set of fiducial Higgs boson cross sections are presented in the context of the simplified template cross section framework. Simplified template cross-sections define fiducial regions in the phase space of Higgs boson production, minimising their dependence on underlying theoretical assumptions.

\section{High-Luminosity LHC prospects}

The LHC will undergo a major upgrade in the 2020 to restart in the High Luminosity phase (HL-LHC) at $14 \mathrm{TeV}$ center-of-mass energy and with a factor of 10 luminosity increase beyond 

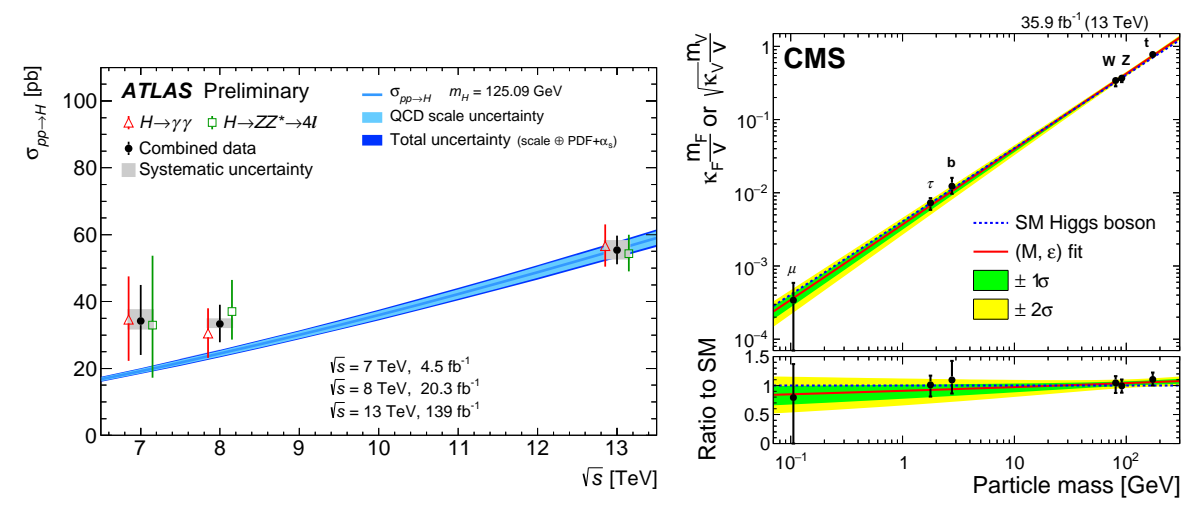

Figure 5: [left:] Total $p p \rightarrow H+X$ cross sections measured at center-of-mass energies of 7, 8 , and $13 \mathrm{TeV}$, compared to Standard Model predictions [24]. Results of the phenomenological fit of the Higgs couplings modifiers within the $\kappa$-framework model [25].

the LHC's design value. It will collect about $3 \mathrm{ab}^{-1}$ of integrated luminosity at pile-up of up to $\langle\mu\rangle=200$. This large dataset will allow to explore the known particle physics landscape with unprecedent precision and will extend the sensitivity to possible anomalies in the Standard Model [27]. The high pile-up will pose major challenges for the experiments. Many upgrades are planned to improve radiation hardness, replace the ageing detectors and provide handles to mitigate pileup and maintain trigger acceptance. The HL-LHC is expected to reach a $4 \mathrm{MeV}$ precision on $m_{W}$, thanks in particular to a 30\% reduction of PDF uncertainty with extended tracking acceptance. For $\sin ^{2} \theta_{\text {eff }}^{l}$ a $15 \cdot 10^{-5}$ uncertainty can be achieved at each experiment (including LHCb). Measurements of the top mass will reach $\sim 200 \mathrm{MeV}$ precision for determinations from direct reconstruction, and indirect determinations will go well below the $\mathrm{GeV}$, posing high challenges to theory. An uncertainty of $20 \mathrm{MeV}$ is expected on the Higgs mass, while, assuming theory uncertainties can be reduced by a factor of two, the Higgs coupling structure will be probed to a few $\%$ precision.

\section{Summary}

Experiments at the LHC are reaching the highest precision for single-experiment measurements of SM parameters, including a first measurement of the $W$ boson mass. A major limiting factor in the precision of these measurements are the uncertainties coming from the accuracy of the theoretical modelling and the limited knowledge of the proton structure. To reach the experimental precision goal of the LHC Run-3 and the future HL-LHC, significant theoretical progress is needed to further reduce theoretical uncertainties.

\section{References}

[1] L. Evans and P. Bryant (editors), 2008 JINST 3 S08001.

[2] ATLAS Collaboration, 2008 JINST 3 S08003.

[3] CMS Collaboration, 2008 JINST 3 S08004. 
[4] GFitter Collaboration, EPJC 78 (2018) 675.

[5] ATLAS Collaboration, EPJC 78 (2018) 110.

[6] ATLAS Collaboration, JHEP 09 (2014) 145.

[7] CMS Collaboration, CMS-PAS-SMP-14-007, https://cds.cern.ch/record/2139655.

[8] CMS Collaboration, JHEP 02 (2017) 096.

[9] ATLAS Collaboration, ATL-PHYS-PUB-2017-021, https://cds.cern.ch/record/2298152.

[10] ATLAS Collaboration, EPJC 77 (2017) 367.

[11] ATLAS Collaboration, ATL-PHYS-PUB-2019-016, https://cds.cern.ch/record/2670662/.

[12] CMS Collaboration, JHEP 12 (2019) 059.

[13] CMS Collaboration, EPJC 78 (2018) 701.

[14] ATLAS Collaboration, JHEP 08 (2016) 159.

[15] ATLAS Collaboration, ATL-CONF-2018-037, https://cds.cern.ch/record/2630340

[16] https://twiki.cern.ch/twiki/bin/view/LHCPhysics/LHCTopWGSummaryPlots

[17] ATLAS Collaboration, arXiv:1910.08819.

[18] CMS Collaboration, EPJC 79 (2019) 313.

[19] ATLAS Collaboration, arXiv:1905.02302.

[20] CMS Collaboration, arXiv:1904.05237.

[21] ATLAS and CMS Collaborations, PRL 114 (2015) 191803

[22] ATLAS Collaboration, PLB 784 (2018) 345.

[23] CMS Collaboration, JHEP 11 (2017) 047.

[24] ATLAS Collaboration, ATLAS-CONF-2019-032, http://cdsweb.cern.ch/record/2682844

[25] CMS Collaboration, EPJC 79 (2019) 421.

[26] ATLAS Collaboration, PRD 101, 012002 (2020).

[27] arXiv:1902.00134, arXiv:1902.04070 